\title{
Quasi-Spherical and Multi-Quasi-Spherical Polynomial Quaternionic Equations: Introduction of the Notions and Some Examples
}

\author{
Dmytro Mierzejewski ${ }^{1}$
}

\begin{abstract}
We introduce notions of quasi-spherical and multi-quasispherical polynomial quaternionic equations defined in terms of the shape of the set of the solutions of the equation. We establish that every quaternionic equation of the form $\sum_{\ell=1}^{m} a^{(\ell)} x^{2} b^{(\ell)}+\sum_{p=1}^{q} c^{(p)} x d^{(p)}+h=0$ is quasi-spherical. We get some sufficient conditions under which a quadratic quaternionic polynomial can be represented as a product of linear quaternionic polynomials (and thus the corresponding equation is multiquasi-spherical).
\end{abstract}

Mathematics Subject Classification (2010). 11R52, 30G35, 12 K99.

Keywords. Quaternion, equation, polynomial, section by a hyperplane, factorization, system of equations.

\section{Introduction}

Since the system of quaternions is the most known extension of the system of complex numbers, it is natural to investigate solutions of polynomial quaternionic equations (by other words, zeros of quaternionic polynomials). But this task is very difficult if one tries to investigate general polynomial quaternionic equation (even of a certain degree). Due to non-commutativity of quaternions, the general form of a polynomial quaternionic equation is

$$
\sum_{p=1}^{n}\left(\sum_{\ell=1}^{m_{p}} a_{p, \ell, 1} x a_{p, \ell, 2} x \ldots a_{p, \ell, p} x a_{p, \ell, p+1}\right)+c=0 .
$$

\footnotetext{
${ }^{1}$ Partially supported by State Foundation of Fundamental Researches of Ukraine (project no. $25.1 / 084)$.
} 
The most deeply investigated polynomial quaternionic equations are socalled classical ones, i.e., of the form

$$
\sum_{p=0}^{n} a_{p} x^{p}=0
$$

or

$$
\sum_{p=0}^{n} x^{p} a_{p}=0 .
$$

Equations of this kind were investigated, e. g., in [5], [4], [3].

Several types of other (not classical) polynomial quaternionic equations were investigated, e. g., in [1], [6], [4], [2], [7].

Unlike real and complex polynomial equations, quaternionic ones often have infinitely many solutions. E. g., according to [5], the set of the solutions of any classical polynomial quaternionic equation consists of spheres and isolated points (sometimes only the spheres or only the points are involved). (Of course, we use the natural interpretation of quaternions as points of the 4-dimensional space.) According to [6], the set of the solutions of any linear quaternionic equation is a linear manifold (or, maybe, the empty set). In [4], [2] again isolated points, spheres and also circles are referred to. In [7] many other types of sets arise in examples.

In this paper we want to define a class of polynomial quaternionic equations whose sets of the solutions have more or less simple shape. We will name such equations quasi-spherical. In order to introduce a corresponding definition let us take into account the following.

Note firstly that we will describe sets of solutions considering their sections by hyperplanes perpendicular to the real axis (as in Section 4 of [2]). By other words, for an equation we will study the sets of its solutions having the same real component.

Then, let us note that spheres are referred to several times above. Take this surface as a basic notion to decide which equations should be called quasi-spherical. The 1-dimensional analogue of a sphere is a circle; the 0dimensional one is a set of two points. One point can be treated as a sphere with zero radius. Imagining a sphere or a circle with infinite radius one obtains respectively a plane or a straight line. At last, a 3-dimensional sphere (i.e., the border of a 4-dimensional ball) degenerates into a 3-dimensional space (a hyperplane of the 4-dimensional space) when its radius turns into infinity.

So, we will call quasi-spherical any polynomial quaternionic equation whose set of the solutions has the following property: every one of its sections by a hyperplane perpendicular to the real axis is one of the figures whose names are written by the bold type in the previous paragraph.

Besides we will call multi-quasi-spherical any polynomial quaternionic equation whose set of the solutions is a finite union of sets suitable for quasispherical ones.

It is interesting to note that in fact polynomial quaternionic equations are multi-quasi-spherical very often. Moreover, up to now the author does 
not know whether there exists at least one polynomial quaternionic equation being not multi-quasi-spherical. It would be a reasonable hypothesis that every polynomial quaternionic equation is multi-quasi-spherical (or, maybe, with a restriction on the degree). ${ }^{2}$

In this paper we establish that quadratic quaternionic equations of a certain kind are quasi-spherical. Then we establish that quadratic quaternionic equations of another certain kind are multi-quasi-spherical, and simultaneously we describe a method to solve equations of this kind.

\section{Preliminaries}

We use standard notations $i, j, k$ for the quaternionic imaginary units; recall that $i^{2}=j^{2}=k^{2}=-1, i j=-j i=k, j k=-k j=i, k i=-i k=j$. Below we will always use subscripts to denote the components of a quaternion as follows: $\xi=\xi_{0}+\xi_{1} i+\xi_{2} j+\xi_{3} k$, where $\xi_{0}, \xi_{1}, \xi_{2}, \xi_{3} \in \mathbb{R}$.

In every equation of this paper the letter $x$ denotes the unknown and other letters denote given coefficients, if there is no other explanation. We name a quaternionic equation any one in which every coefficient is a quaternion. As for solutions of such equations, we always consider solutions being quaternions. Analogously, a polynomial in which every coefficient is a quaternion will be called a quaternionic polynomial; treating a quaternionic polynomial as a function we will consider that it is a function of quaternionic variable.

Definition 1. Let a quaternionic equation of the form (1) be given. Fix a certain $\xi_{0} \in \mathbb{R}$ and consider the set $S\left(\xi_{0}\right)$ of solutions $x$ of the given equation such that $x_{0}=\xi_{0}$. Let for each $\xi_{0} \in \mathbb{R} S\left(\xi_{0}\right)$ be either the empty set, or a point, or a set of two points, or a straight line, or a circle, or a plane, or a sphere, or the entire hyperplane $x_{0}=\xi_{0}$. Then the given equation is called a quasi-spherical polynomial quaternionic equation.

Definition 2. Let a quaternionic equation of the form (1) be given. Fix a certain $\xi_{0} \in \mathbb{R}$ and consider the set $S\left(\xi_{0}\right)$ of solutions $x$ of the given equation such that $x_{0}=\xi_{0}$. Let for each $\xi_{0} \in \mathbb{R} S\left(\xi_{0}\right)$ be either the empty set, or the entire hyperplane $x_{0}=\xi_{0}$, or a finite union of sets each from them is either a point, or a straight line, or a circle, or a plane, or a sphere. Then the given equation is called a multi-quasi-spherical polynomial quaternionic equation.

Obviously, every quasi-spherical polynomial quaternionic equation is also multi-quasi-spherical one (of course, we treat any set as a "union of this one set").

Now we will formulate two propositions following from previous papers on this topic.

Proposition 1. Every quaternionic equation of the form (2) or (3) (i.e., classical one) is quasi-spherical.

\footnotetext{
${ }^{2}$ After submitting this paper the author has found out that the hypothesis is wrong: $i x i x+x i x i=0$ is a quadratic quaternionic equation being not multi-quasi-spherical.
} 
This fact follows from [5]. More exactly, according to [5], each above mentioned section of the set of the solutions is either a sphere, or a point, or the empty set.

Proposition 2. Every quaternionic equation of the form

$$
\sum_{\ell=1}^{m} a^{(\ell)} x b^{(\ell)}+c=0
$$

(i.e., linear one) is quasi-spherical.

This fact follows from [6] where it was established that the set of the solutions of such equation is a linear manifold (or, maybe, the empty set). Obviously, any section of a linear manifold by a hyperplane is also a linear manifold: either a hyperplane, or a plane, or a line, or a point (or the empty set if the hyperplane does not intersect the manifold).

\section{Quadratic Quaternionic Equations with Non-Split Squares}

Definition 3. A quadratic quaternionic equation is called one with non-split squares if it is of the following form:

$$
\sum_{\ell=1}^{m} a^{(\ell)} x^{2} b^{(\ell)}+\sum_{p=1}^{q} c^{(p)} x d^{(p)}+h=0 .
$$

Now we will establish a result generalizing the main result of Section 4 of $[2]$.

Proposition 3. Every quadratic quaternionic equation with non-split squares is quasi-spherical.

Proof. Let us pass from (4) to the corresponding system of real equations. For this aim one has to rewrite $x$ as $x_{0}+x_{1} i+x_{2} j+x_{3} k$ and analogously every coefficient, to commit multiplications and to get an equation with real functions $f_{0}, f_{1}, f_{2}, f_{3}$ :

$$
\begin{gathered}
f_{0}\left(x_{0}, x_{1}, x_{2}, x_{3}\right)+f_{1}\left(x_{0}, x_{1}, x_{2}, x_{3}\right) i+ \\
f_{2}\left(x_{0}, x_{1}, x_{2}, x_{3}\right) j+f_{3}\left(x_{0}, x_{1}, x_{2}, x_{3}\right) k=0 .
\end{gathered}
$$

This equation is equivalent to the following system of four real equations:

$$
\left\{\begin{array}{l}
f_{0}\left(x_{0}, x_{1}, x_{2}, x_{3}\right)=0 \\
f_{1}\left(x_{0}, x_{1}, x_{2}, x_{3}\right)=0 \\
f_{2}\left(x_{0}, x_{1}, x_{2}, x_{3}\right)=0 \\
f_{3}\left(x_{0}, x_{1}, x_{2}, x_{3}\right)=0
\end{array}\right.
$$

Of course, it is possible to write down (5) in detail, but it is very complicated and not necessary for the proof. It will be sufficient for us to establish only several properties of the expressions $f_{r}\left(x_{0}, x_{1}, x_{2}, x_{3}\right)$. Let us investigate contribution of each term of (4) to the expressions $f_{r}\left(x_{0}, x_{1}, x_{2}, x_{3}\right)$.

First of all, it is easy to understand that the terms of the form $c^{(p)} x d^{(p)}$ and $h$ generate only linear expressions with respect to $x_{0}, x_{1}, x_{2}, x_{3}$. 
Then consider any term of the form $a^{(\ell)} x^{2} b^{(\ell)}$. Its contribution to each $f_{r}\left(x_{0}, x_{1}, x_{2}, x_{3}\right)$ can be found by transformation of the following expression: $\left(a_{0}^{(\ell)}+a_{1}^{(\ell)} i+a_{2}^{(\ell)} j+a_{3}^{(\ell)} k\right)\left(x_{0}+x_{1} i+x_{2} j+x_{3} k\right)^{2}\left(b_{0}^{(\ell)}+b_{1}^{(\ell)} i+b_{2}^{(\ell)} j+b_{3}^{(\ell)} k\right)$.

Observe that the square generates terms with only the following products of $x_{s}: x_{0}^{2}, x_{1}^{2}, x_{2}^{2}, x_{3}^{2}, x_{0} x_{1}, x_{0} x_{2}, x_{0} x_{3}$.

We have to consider $x_{0}$ as a fixed number. Therefore the above mentioned products $x_{0} x_{1}, x_{0} x_{2}, x_{0} x_{3}$ can be treated as linear expressions. Taking into account all just explained, it is not difficult to establish that the part of any $f_{r}\left(x_{0}, x_{1}, x_{2}, x_{3}\right)$, generated by $a^{(\ell)} x^{2} b^{(\ell)}$, looks as follows:

$$
K\left(x_{1}^{2}+x_{2}^{2}+x_{3}^{2}\right)+L\left(x_{0} ; x_{1}, x_{2}, x_{3}\right),
$$

where $K$ is a real constant and $L\left(x_{0} ; x_{1}, x_{2}, x_{3}\right)$ is an expression linear with respect to $x_{1}, x_{2}, x_{3}$ (but no linearity if one considers also $x_{0}$ as an argument).

We have already investigated the contribution of every term from (4). So, each $f_{r}\left(x_{0}, x_{1}, x_{2}, x_{3}\right)$ is a sum of $m$ expressions of the form (6) and $q+1$ expressions linear with respect to $x_{0}, x_{1}, x_{2}, x_{3}$. Obviously, this sum is again of the form (6).

Now we can rewrite (5) as follows:

$$
\left\{\begin{array}{l}
K_{0}\left(x_{1}^{2}+x_{2}^{2}+x_{3}^{2}\right)+L_{0}\left(x_{0} ; x_{1}, x_{2}, x_{3}\right)=0 \\
K_{1}\left(x_{1}^{2}+x_{2}^{2}+x_{3}^{2}\right)+L_{1}\left(x_{0} ; x_{1}, x_{2}, x_{3}\right)=0 \\
K_{2}\left(x_{1}^{2}+x_{2}^{2}+x_{3}^{2}\right)+L_{2}\left(x_{0} ; x_{1}, x_{2}, x_{3}\right)=0 \\
K_{3}\left(x_{1}^{2}+x_{2}^{2}+x_{3}^{2}\right)+L_{3}\left(x_{0} ; x_{1}, x_{2}, x_{3}\right)=0
\end{array}\right.
$$

where each $L_{r}\left(x_{0} ; x_{1}, x_{2}, x_{3}\right)$ is a linear expression with respect to $x_{1}, x_{2}, x_{3}$. If $x_{0}$ is fixed then each equation from (7) represents a sphere, or a linear manifold, or the empty set. Obviously, the intersection of four such sets gives just a set from those mentioned in the definition of a quasi-spherical equation, and the proposition is proved.

\section{Factorable Quadratic Quaternionic Equations}

Consider a polynomial quaternionic equation of the form

$$
P(x) Q(x)=0,
$$

where $P$ and $Q$ are quaternionic polynomials (and neither $P$ nor $Q$ is a constant). Of course, due to absence of zero divisors in the system of quaternions, the set of the solutions of (8) is the union of the sets of the solutions of the equations $P(x)=0$ and $Q(x)=0$. So, factorization of the left-hand side of a polynomial quaternionic equation may help to obtain information about its solutions.

Nevertheless for polynomial quaternionic equations connection between solutions and factorization is not so simple as for complex ones. For example, the equation $x^{2}-i=0$ has exactly two quaternionic solutions: $\frac{1+i}{\sqrt{2}}$ and $-\frac{1+i}{\sqrt{2}}$ (this fact can be checked by the theory from [5]). But the expressions $x^{2}-i$ and $\left(x-\frac{1+i}{\sqrt{2}}\right)\left(x+\frac{1+i}{\sqrt{2}}\right)$ are not identically equal if $x$ is any quaternion; the 
latter expression equals $x^{2}+\frac{x i}{\sqrt{2}}-\frac{i x}{\sqrt{2}}-i$ and cannot be reduced to the former expression due to non-commutativity of quaternions.

So, an important question about a given quaternionic equation is whether it can be written in the form (8). Such factorization is especially useful if it is known a convenient method to solve the equations $P(x)=0$ and $Q(x)=0$. We will investigate the case where these equations are linear. Any linear quaternionic equation can be comparatively easily solved by passing to a system of real equations. Moreover, according to Proposition 2, any linear quaternionic equation is quasi-spherical and therefore the equation (8) is multi-quasi-spherical in this case.

Now our aim is to find conditions under which a quadratic equation can be represented in the form (8) with linear $P$ and $Q$. For a certain particular form of $P$ and $Q$ this problem was solved in Section 3 of [2]. Now we consider the general form of linear quaternionic polynomials: $P(x)=\sum_{\ell=1}^{m} \alpha^{(\ell)} x \beta^{(\ell)}+a$, $Q(x)=\sum_{p=1}^{q} \gamma^{(p)} x \delta^{(p)}+b$, where there is no zero among the coefficients $\alpha^{(1)}$, $\ldots, \alpha^{(m)}, \beta^{(1)}, \ldots, \beta^{(m)}, \gamma^{(1)}, \ldots, \gamma^{(q)}, \delta^{(1)}, \ldots, \delta^{(q)}$.

Rewrite the product of the linear polynomials as follows:

$$
\begin{array}{r}
\left(\sum_{\ell=1}^{m} \alpha^{(\ell)} x \beta^{(\ell)}+a\right)\left(\sum_{p=1}^{q} \gamma^{(p)} x \delta^{(p)}+b\right)= \\
\sum_{\ell=1}^{m}\left(\sum_{p=1}^{q} \alpha^{(\ell)} x \beta^{(\ell)} \gamma^{(p)} x \delta^{(p)}\right)+\sum_{\ell=1}^{m} \alpha^{(\ell)} x \beta^{(\ell)} b+\sum_{p=1}^{q} a \gamma^{(p)} x \delta^{(p)}+a b .
\end{array}
$$

Setting $\beta^{(\ell)} \gamma^{(p)}=: r^{(\ell, p)}, \beta^{(\ell)} b=: s^{(\ell)}, a \gamma^{(p)}=: u^{(p)}, a b=: v$, we obtain the following form of a quadratic quaternionic equation for which it is possible to hope that it is factorable:

$$
\sum_{\ell=1}^{m}\left(\sum_{p=1}^{q} \alpha^{(\ell)} x r^{(\ell, p)} x \delta^{(p)}\right)+\sum_{\ell=1}^{m} \alpha^{(\ell)} x s^{(\ell)}+\sum_{p=1}^{q} u^{(p)} x \delta^{(p)}+v=0 .
$$

Note that we already see many restrictions on such equations. Two natural numbers $m$ and $q$ have to exist such that the number of quadratic terms in the equation would equal $m q$ and the number of its linear terms would equal $m+q$. Moreover equal coefficients in certain positions have to be present. Therefore this class of equations is, unfortunately, very narrow.

Given an equation of the form (9) and trying to factor it, one has to solve the following system of quaternionic equations: $\forall \ell \in\{1, \ldots, m\}, \forall p \in\{1, \ldots, q\}$

$$
\left\{\begin{array}{l}
\beta^{(\ell)} \gamma^{(p)}=r^{(\ell, p)}, \\
\beta^{(\ell)} b=s^{(\ell)} \\
a \gamma^{(p)}=u^{(p)} \\
a b=v
\end{array}\right.
$$


where the unknowns are $a, b, \beta^{(1)}, \ldots, \beta^{(m)}, \gamma^{(1)}, \ldots, \gamma^{(q)}$. If this system is solvable then the equation is factorable.

Note firstly that, since we consider the case where any $\beta^{(\ell)}$ and any $\gamma^{(p)}$ does not equal zero, we should also assume that any $r^{(\ell, p)}$ does not equal zero (by the way, otherwise the equation is not quadratic). Moreover let us restrict our considerations investigating only the case where $v \neq 0$, $\forall \ell \in\{1, \ldots, m\} \quad s^{(\ell)} \neq 0, \forall p \in\{1, \ldots, q\} \quad u^{(p)} \neq 0$. Then, obviously, any solution of the system cannot contain zero. Under these conditions (10) can be rewritten as follows:

$$
\left\{\begin{array}{l}
\gamma^{(p)}=\left(\beta^{(\ell)}\right)^{-1} r^{(\ell, p)} \\
\beta^{(\ell)}=s^{(\ell)} b^{-1} \\
\gamma^{(p)}=a^{-1} u^{(p)} \\
b=a^{-1} v
\end{array}\right.
$$

Then we perform the following equivalent transformations of the system:

$$
\begin{aligned}
& \left\{\begin{array}{l}
\gamma^{(p)}=\left(\beta^{(\ell)}\right)^{-1} r^{(\ell, p)}, \\
\beta^{(\ell)}=s^{(\ell)} v^{-1} a, \\
\gamma^{(p)}=a^{-1} u^{(p)} \\
b=a^{-1} v
\end{array}\right. \\
& \left\{\begin{array}{l}
\gamma^{(p)}=a^{-1} v\left(s^{(\ell)}\right)^{-1} r^{(\ell, p)}, \\
\beta^{(\ell)}=s^{(\ell)} v^{-1} a, \\
\gamma^{(p)}=a^{-1} u^{(p)} \\
b=a^{-1} v
\end{array}\right.
\end{aligned}
$$

Now it is obvious that the system is solvable if and only if $\forall \ell \in\{1, \ldots, m\}$, $\forall p \in\{1, \ldots, q\}$

$$
u^{(p)}=v\left(s^{(\ell)}\right)^{-1} r^{(\ell, p)} .
$$

Moreover if (12) holds true then in order to factor the equation (9) it is possible to take any non-zero quaternion $a$ and to calculate other unknown coefficients by the following formulas (implied by (11)): $\forall \ell \in\{1, \ldots, m\}$, $\forall p \in\{1, \ldots, q\}$

$$
b=a^{-1} v, \quad \beta^{(\ell)}=s^{(\ell)} v^{-1} a, \quad \gamma^{(p)}=a^{-1} u^{(p)} .
$$

Of course, the best way is to put $a=1$. Then instead of (13) we get: $\forall \ell \in$ $\{1, \ldots, m\}, \forall p \in\{1, \ldots, q\}$

$$
a=1, \quad b=v, \quad \beta^{(\ell)}=s^{(\ell)} v^{-1}, \quad \gamma^{(p)}=u^{(p)} .
$$

As a result of the above considerations we obtain the following proposition:

Proposition 4. Let a quaternionic equation of the following form be given:

$$
\sum_{\ell=1}^{m}\left(\sum_{p=1}^{q} \alpha^{(\ell)} x r^{(\ell, p)} x \delta^{(p)}\right)+\sum_{\ell=1}^{m} \alpha^{(\ell)} x s^{(\ell)}+\sum_{p=1}^{q} u^{(p)} x \delta^{(p)}+v=0,
$$


where no coefficient equals zero. Besides let the following condition hold true: $\forall \ell \in\{1, \ldots, m\}, \forall p \in\{1, \ldots, q\}$

$$
u^{(p)}=v\left(s^{(\ell)}\right)^{-1} r^{(\ell, p)} .
$$

Then this equation is multi-quasi-spherical and can be rewritten as follows:

$$
\left(\sum_{\ell=1}^{m} \alpha^{(\ell)} x s^{(\ell)} v^{-1}+1\right)\left(\sum_{p=1}^{q} u^{(p)} x \delta^{(p)}+v\right)=0 .
$$

Then it is possible to widen this class of factorable equations by the following considerations. It is known that every real number commutes with every quaternion. Therefore in (9) it is possible to move real factors between different quaternionic coefficients in the same term. Thus for the factorability it is enough to be able to obtain an equation satisfying (12) after some above mentioned rearrangement of real factors.

Let us investigate in detail how to pass from (9) to another factorable quaternionic equation of the form

$$
\sum_{\ell=1}^{m}\left(\sum_{p=1}^{q} \tilde{\alpha}^{(\ell)} x \tilde{r}^{(\ell, p)} x \tilde{\delta}^{(p)}\right)+\sum_{\ell=1}^{m} \tilde{\tilde{\alpha}}^{(\ell)} x \tilde{s}^{(\ell)}+\sum_{p=1}^{q} \tilde{u}^{(p)} x \tilde{\tilde{\delta}}^{(p)}+v=0 .
$$

(Again we consider that any coefficient of (15) does not equal zero). For each $\ell \in\{1, \ldots, m\}$ and for each $p \in\{1, \ldots, q\}$ non-zero real numbers $\rho^{(1, \ell)}, \rho^{(2, p)}$, $\sigma^{(\ell)}, \tau^{(p)}$ should exist such that $\forall \ell \in\{1, \ldots, m\}, \forall p \in\{1, \ldots, q\}$

$$
\begin{aligned}
& \tilde{\alpha}^{(\ell)}=\rho^{(1, \ell)} \alpha^{(\ell)}, \quad \tilde{\delta}^{(p)}=\rho^{(2, p)} \delta^{(p)}, \quad \tilde{r}^{(\ell, p)}=\frac{r^{(\ell, p)}}{\rho^{(1, \ell)} \rho^{(2, p)}} ; \\
& \tilde{\tilde{\alpha}}^{(\ell)}=\sigma^{(\ell)} \alpha^{(\ell)}, \quad \tilde{s}^{(\ell)}=\frac{s^{(\ell)}}{\sigma^{(\ell)}} ; \\
& \tilde{u}^{(p)}=\tau^{(p)} u^{(p)}, \quad \tilde{\tilde{\delta}}(p)=\frac{\delta^{(p)}}{\tau^{(p)}} .
\end{aligned}
$$

These conditions imply that $\forall \ell \in\{1, \ldots, m\}, \forall p \in\{1, \ldots, q\}$

$$
\tilde{\tilde{\alpha}}^{(\ell)}=\frac{\sigma^{(\ell)}}{\rho^{(1, \ell)}} \tilde{\alpha}^{(\ell)}, \quad \tilde{\tilde{\delta}}(p)=\frac{\tilde{\delta}^{(p)}}{\rho^{(2, p)} \tau^{(p)}} .
$$

Putting $\frac{\sigma^{(\ell)}}{\rho^{(1, \ell)}}=: \mu^{(\ell)}, \frac{1}{\rho^{(2, p)} \tau^{(p)}}=: \nu^{(p)}$ we obtain the following easily checkable condition: $\forall \ell \in\{1, \ldots, m\}, \forall p \in\{1, \ldots, q\}$

$$
\tilde{\tilde{\alpha}}^{(\ell)}=\mu^{(\ell)} \tilde{\alpha}^{(\ell)}, \quad \tilde{\tilde{\delta}}(p)=\nu^{(p)} \tilde{\delta}^{(p)},
$$

where each $\mu^{(\ell)}$ and each $\nu^{(p)}$ may be any non-zero real number. It is convenient to rewrite the above formulas in terms of these $\mu^{(\ell)}$ and $\nu^{(p)}$. Therefore we represent $\sigma^{(\ell)}$ and $\tau^{(p)}$ as follows: $\sigma^{(\ell)}=\mu^{(\ell)} \rho^{(1, \ell)}, \tau^{(p)}=\frac{1}{\nu^{(p)} \rho^{(2, p)}}$. Then we have instead of $(16): \forall \ell \in\{1, \ldots, m\}, \forall p \in\{1, \ldots, q\}$

$$
\begin{gathered}
\tilde{\alpha}^{(\ell)}=\rho^{(1, \ell)} \alpha^{(\ell)}, \quad \tilde{\delta}^{(p)}=\rho^{(2, p)} \delta^{(p)}, \quad \tilde{r}^{(\ell, p)}=\frac{r^{(\ell, p)}}{\rho^{(1, \ell)} \rho^{(2, p)}} ; \\
\tilde{\tilde{\alpha}}^{(\ell)}=\mu^{(\ell)} \rho^{(1, \ell)} \alpha^{(\ell)}, \quad \tilde{s}^{(\ell)}=\frac{s^{(\ell)}}{\mu^{(\ell)} \rho^{(1, \ell)}} ; \\
\tilde{u}^{(p)}=\frac{u^{(p)}}{\nu^{(p)} \rho^{(2, p)}}, \quad \tilde{\tilde{\delta}}(p)=\nu^{(p)} \rho^{(2, p)} \delta^{(p)}
\end{gathered}
$$


or, in a form being more convenient for calculations below: $\forall \ell \in\{1, \ldots, m\}$, $\forall p \in\{1, \ldots, q\}$

$$
\begin{gathered}
\alpha^{(\ell)}=\frac{\tilde{\alpha}^{(\ell)}}{\rho^{(1, \ell)}}, \quad \delta^{(p)}=\frac{\tilde{\delta}^{(p)}}{\rho^{(2, p)}}, \quad r^{(\ell, p)}=\rho^{(1, \ell)} \rho^{(2, p)} \tilde{r}^{(\ell, p)} ; \\
\alpha^{(\ell)}=\frac{\tilde{\tilde{\alpha}}^{(\ell)}}{\mu^{(\ell)} \rho^{(1, \ell)}}, \quad s^{(\ell)}=\mu^{(\ell)} \rho^{(1, \ell)} \tilde{s}^{(\ell)} \\
u^{(p)}=\nu^{(p)} \rho^{(2, p)} \tilde{u}^{(p)}, \quad \delta^{(p)}=\frac{\tilde{\tilde{\delta}}^{(p)}}{\nu^{(p)} \rho^{(2, p)}} .
\end{gathered}
$$

From (12) and (18) we obtain: $\forall \ell \in\{1, \ldots, m\}, \forall p \in\{1, \ldots, q\}$

$$
\mu^{(\ell)} \nu^{(p)} \tilde{u}^{(p)}=v\left(\tilde{s}^{(\ell)}\right)^{-1} \tilde{r}^{(\ell, p)} .
$$

Then from (14) and (18) we obtain: $\forall \ell \in\{1, \ldots, m\}, \forall p \in\{1, \ldots, q\}$

$$
\begin{gathered}
a=1, \quad b=v, \quad \alpha^{(\ell)}=\frac{\tilde{\alpha}^{(\ell)}}{\rho^{(1, \ell)}}, \quad \beta^{(\ell)}=\mu^{(\ell)} \rho^{(1, \ell)} \tilde{s}^{(\ell)} v^{-1}, \\
\gamma^{(p)}=\nu^{(p)} \rho^{(2, p)} \tilde{u}^{(p)}, \quad \delta^{(p)}=\frac{\tilde{\delta}^{(p)}}{\rho^{(2, p)}} .
\end{gathered}
$$

It is easy to see that the choice of the real constants $\rho^{(1, \ell)}$ and $\rho^{(2, p)}$ is not determined by a given equation. Therefore it is possible to put

$$
\rho^{(1,1)}=\cdots=\rho^{(1, m)}=\rho^{(2,1)}=\cdots=\rho^{(2, q)}=1
$$

and to rewrite (20) as follows:

$$
\begin{gathered}
a=1, \quad b=v, \quad \alpha^{(\ell)}=\tilde{\alpha}^{(\ell)}, \quad \beta^{(\ell)}=\mu^{(\ell)} \tilde{s}^{(\ell)} v^{-1}, \\
\gamma^{(p)}=\nu^{(p)} \tilde{u}^{(p)}, \quad \delta^{(p)}=\tilde{\delta}^{(p)} .
\end{gathered}
$$

As a result we obtain the following proposition:

Proposition 5. Let a quaternionic equation of the following form be given:

$$
\sum_{\ell=1}^{m}\left(\sum_{p=1}^{q} \tilde{\alpha}^{(\ell)} x \tilde{r}^{(\ell, p)} x \tilde{\delta}^{(p)}\right)+\sum_{\ell=1}^{m} \tilde{\tilde{\alpha}}^{(\ell)} x \tilde{s}^{(\ell)}+\sum_{p=1}^{q} \tilde{u}^{(p)} x \tilde{\tilde{\delta}}^{(p)}+v=0,
$$

where no coefficient equals zero and there exist some real numbers $\mu^{(1)}, \ldots$, $\mu^{(m)}, \nu^{(1)}, \ldots, \nu^{(q)}$ such that $\forall \ell \in\{1, \ldots, m\}, \forall p \in\{1, \ldots, q\}$

$$
\tilde{\tilde{\alpha}}^{(\ell)}=\mu^{(\ell)} \tilde{\alpha}^{(\ell)}, \quad \tilde{\tilde{\delta}}(p)=\nu^{(p)} \tilde{\delta}^{(p)} .
$$

Besides let the following condition hold true: $\forall \ell \in\{1, \ldots, m\}, \forall p \in\{1, \ldots, q\}$

$$
\mu^{(\ell)} \nu^{(p)} \tilde{u}^{(p)}=v\left(\tilde{s}^{(\ell)}\right)^{-1} \tilde{r}^{(\ell, p)} .
$$

Then this equation is multi-quasi-spherical and can be rewritten as follows:

$$
\left(\sum_{\ell=1}^{m} \tilde{\alpha}^{(\ell)} x \mu^{(\ell)} \tilde{s}^{(\ell)} v^{-1}+1\right)\left(\sum_{p=1}^{q} \nu^{(p)} \tilde{u}^{(p)} x \tilde{\delta}^{(p)}+v\right)=0 .
$$

At last recall that we have investigated the case where $v \neq 0, \forall \ell \in$ $\{1, \ldots, m\} s^{(\ell)} \neq 0, \forall p \in\{1, \ldots, q\} u^{(p)} \neq 0$. Other cases can be investigated similarly. Such investigation may be offered as a useful exercise for a student.

\section{Acknowledgements}

The author is thankful to V. Szpakowski for useful discussions. 


\section{References}

[1] S. Eilenberg, I. Niven, The "fundamental theorem of algebra" for quaternions. Bulletin of the American Mathematical Society. - 1944, vol. 50, no. 4, pp. 246248.

[2] D. Mierzejewski, Investigation of quaternionic quadratic equations I. Factorization and passing to a system of real equations. Bulletin de la Société des Sciences et des Lettres de Łódź. Série: Recherches sur les Déformations. - 2008, vol. 56, pp. 17-26.

[3] D. Mierzejewski, Investigation of quaternionic quadratic equations II. Method of solving an equation with left coefficients. Bulletin de la Société des Sciences et des Lettres de Eódź. Série: Recherches sur les Déformations. - 2008, vol. 57, pp. 19-24.

[4] D. Mierzejewski, V. Szpakowski, On solutions of some types of quaternionic quadratic equations. Bulletin de la Société des Sciences et des Lettres de Eódź. Série: Recherches sur les Déformations. - 2008, vol. 55, pp. 49-58.

[5] A. Pogorui, M. Shapiro, On the structure of the set of the zeros of quaternionic polynomials. Complex Variables and Elliptic Equations. - 2004, vol. 49, no. 6, pp. 379-389.

[6] V. Szpakowski, Solution of general linear quaternionic equations. The XI Kravchuk International Scientific Conference. - Kyiv (Kiev), Ukraine, 2006, p. 661. (In Ukrainian.)

[7] V. Szpakowski, Solution of quadratic quaternionic equations. Bulletin de la Société des Sciences et des Lettres de Łódź. Série: Recherches sur les Déformations. - 2009, vol. 58, pp. 45-58.

Dmytro Mierzejewski

Teatralna Street, 5-b, ap. 6

Zhytomyr, 10014

Ukraine

e-mail: dmytro1972@gmail.com

Received: August 24, 2009.

Accepted: November 3, 2009.

Open Access This article is distributed under the terms of the Creative Commons Attribution Noncommercial License which permits any noncommercial use, distribution, and reproduction in any medium, provided the original author(s) and source are credited. 